\title{
有机膦氧化还原催化反应研究进展
}

\author{
蔡卫黄 有* \\ (南开大学化学学院 元素有机化学国家重点实验室 天津 300071)
}

\begin{abstract}
摘要 有机膦试剂在有机合成中占据非常重要的地位, 有机膦化合物介导的 Wittig 反应、Staudinger 反应、Appel 反应、 Mitsunobu 反应发展至今在科学研究和工业生产等方面意义重大. 近年来关于 $\mathrm{P}(\mathrm{III}) / \mathrm{P}(\mathrm{V})$ 氧化还原循环体系、催化剂的 设计, 实现这些反应的催化模式, 避免计量 $\mathrm{R}_{3} \mathrm{P}=\mathrm{O}$ 副产物的产生的研究快速发展. 本综述从各个反应出发, 依次介绍 这些反应的催化过程的研究进展, 对涉及计量 $\mathrm{R}_{3} \mathrm{P}=\mathrm{O}$ 副产物的反应未来可能的发展方向进行了展望.

关键词 Wittig 反应; Staudinger 反应; Appel 反应; Mitsunobu 反应; 膦催化; $\mathrm{P}(\mathrm{III}) / \mathrm{P}(\mathrm{V})$ 氧化还原催化
\end{abstract}

\section{Advances in Organophosphorus Redox Catalysis}

\author{
Cai, Wei Huang, You* \\ (State Key Laboratory and Institute of Elemento-organic Chemistry, College of Chemistry, \\ Nankai University, Tianjin 300071)
}

\begin{abstract}
Organophosphorus reagents play very important role in organic synthesis. The development of Wittig reaction, Staudinger reaction, Appel reaction and Mitsunobu reaction mediated by phosphine compounds is of great significance in scientific research and industrial production. In recent decades, the research on the design of $\mathrm{P}(\mathrm{III}) / \mathrm{P}(\mathrm{V})$ redox catalysis and catalysts to realize the catalytic mode of these reactions, and avoiding the production of stoichiometric $\mathrm{R}_{3} \mathrm{P}=\mathrm{O}$ has developed rapidly. This review introduces the research progress of the catalytic process of each reaction in turn. The future research direction in this field about the reactions involving stoichiometric $\mathrm{R}_{3} \mathrm{P}=\mathrm{O}$ is prospected.

Keywords Wittig reaction; Staudinger reaction; Appel reaction; Mitsunobu reaction; phosphine catalysis; $\mathrm{P}(\mathrm{III}) / \mathrm{P}(\mathrm{V}) \mathrm{redox}$ catalysis
\end{abstract}

磷元素广泛分布于地壳和生物体中, 它与人类的生 命起源、生产和生活密不可分, 磷元素已经广泛地用到 医药、农药、化肥、材料、天然产物、军工以及有机合 成等领域. 在有机合成中, 三价有机膦化合物可以作为 配体、催化剂、试剂, 在有机合成中成为了不可代替的 角色. 作为试剂, 科学家先后发展了许多基于有机膦试 剂的人名反应, 主要有 Wittig 反应 ${ }^{[1]} 、$ Staudinger 反应 ${ }^{[2]} 、$ Appel 反应 ${ }^{[3]} 、$ Mitsunobu 反应 ${ }^{[4]}$ 等. 这些反应为有机合 成提供了许多实用、有效的新方法. 但这些反应存在一 个主要的缺陷, 就是都需要计量的三价膦试剂, 并产生 计量的 $\mathrm{R}_{3} \mathrm{P}=\mathrm{O}$ 副产物, 原子经济性不高, 给后处理过 程也造成了影响. 因此在反应体系中引入还原剂将 $\mathrm{P}(\mathrm{V})$ 还原为 $\mathrm{P}(\mathrm{III})$, 从而实现这些计量有机膦参与的人
名反应的催化版本十分有价值.

在这些 $\mathrm{P}(\mathrm{III}) / \mathrm{P}(\mathrm{V})$ 氧化还原催化循环中，由于 $\mathrm{P}(\mathrm{III})$ 的还原性以及 $\mathrm{P}(\mathrm{V})$ 的相对稳定性, $\mathrm{P}(\mathrm{III})$ 化合物可以被中 等以上氧化能力的氧化剂氧化 ${ }^{[3,5-7]}$; 反过来, 将 $\mathrm{P}(\mathrm{V})$ 还 原为 $\mathrm{P}(\mathrm{III})$ 是逆热力学过程的 ${ }^{[8-9]}$, 这也是实现这一目标 主要挑战. 关于 $\mathrm{P}(\mathrm{III}) / \mathrm{P}(\mathrm{V})$ 氧化还原循环催化的研究, 近年来取得了可喜的成果, 主要有两种思路: (1)通过使 用硅氢、金属氢化物等还原剂将 $\mathrm{P}(\mathrm{V})=\mathrm{O}$ 键经氢化膦烷 中间体生成 $\mathrm{P}(\mathrm{III})^{[10]}$; (2)由于环状的 $\mathrm{P}(\mathrm{V})=\mathrm{O}$ 相对于非 环状结构的 $\mathrm{P}(\mathrm{V})=\mathrm{O}$ 有更低的还原能垒, 设计特殊骨架 的有机膦催化剂[11]. 本文将依次对这些计量有机膦参与 的人名反应的催化版本以及近期新发展的 $\mathrm{P}(\mathrm{III}) / \mathrm{P}(\mathrm{V})$ 氧 化还原循环催化反应体系进行系统综述.

\footnotetext{
* Corresponding author. E-mail: hyou@nankai.edu.cn

Received June 2, 2021; revised July 1, 2021; published online July 12, 2021.

Dedicated to the 100th anniversary of Chemistry at Nankai University.

Project supported by the National Natural Science Foundation of China (Nos. 21871148, 21672109).

国家自然科学基金(Nos. 21871148, 21672109)资助项目.
} 


$$
\begin{aligned}
& \text { Wittig reaction: } \\
& \mathrm{R}_{3} \mathrm{P}+\mathrm{N}_{3}-\mathrm{R}^{1} \longrightarrow \mathrm{R}_{3} \mathrm{P}=\mathrm{N}_{\mathrm{R}^{1}} \stackrel{\mathrm{H}}{\longrightarrow} \mathrm{R}^{1} \mathrm{NH}_{2}+\mathrm{O}=\mathrm{PR}_{3}
\end{aligned}
$$

$$
\begin{aligned}
& \text { Appel reaction: } \\
& \mathrm{R}_{3} \mathrm{P}+\mathrm{R}^{1} \stackrel{\mathrm{O}}{\stackrel{\mathrm{O}}{\dot{H}}} \mathrm{R}^{2} \stackrel{[\mathrm{X}]}{\longrightarrow} \mathrm{R}_{\mathrm{R}^{1}}^{\stackrel{\mathrm{X}}{\mathrm{X}^{2}}}+\mathrm{O}=\mathrm{PR}_{3}
\end{aligned}
$$

$$
\text { Mitsunobu reaction: }
$$

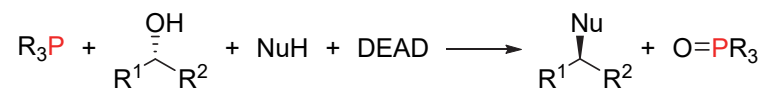

图式 1 计量膦介导的人名反应

Scheme 1 Stoichiometric phosphine mediated name reactions

\section{1 催化 Wittig 反应}

Wittig 反应是最重要的人名反应之一, 利用膦亲氧 的特性高效构建各种各样的烯烃化合物, 它是合成烯烃 的极有价值的一般方法, 在各种重要药物及全合成中的 烯烃的构建, Wittig 反应常常是首选的方法, 但是这一 反应的缺点是大量的 $\mathrm{R}_{3} \mathrm{P}=\mathrm{O}$ 副产物, 往往给产物分离 带来了困扰. 为实现反应中 $\mathrm{P}(\mathrm{III}) / \mathrm{P}(\mathrm{V})$ 氧化还原循环催 化过程, 需要采用温和迅速的策略还原三级氧膦副产 物. 近年来, 通过对有机膦催化剂的设计, 结合计量的 还原剂达到了催化 Wittig 反应的目的.

2009 年, O'Brien 课题组 ${ }^{[12]}$ 发展了首例三级氧膦氧 化还原循环催化 Wittig 反应, 收率中等, $Z / E$ 选择性优秀 (Scheme 2). 反应中使用五元膦杂环氧化物 $\mathbf{P} 1 \cdot[\mathbf{O}]$ 作为 催化剂前体，通过 $\mathrm{Ph}_{2} \mathrm{SiH}_{2}$ 还原使其成为活泼的 $\mathrm{P}(\mathrm{III})$ 物 种，接着与氯代烷烃反应成为季磷盐，再通过去质子 化、Wittig 反应来获得目标产物并再生膦氧化物完成氧 化还原催化循环. 反应有比较好的官能团耐受性, 中等 的收率和较好的 $Z / E$ 选择性. 遗憾的是, 由于膦氧化物 难以还原, 反应需要在较高的温度下进行.

该作者在 2013 年 ${ }^{[13]}$ 发现在反应体系中加入催化量 的对硝基苯甲酸这一布朗斯特酸作添加剂, 可以进一步 改良此氧化还原催化循环反应(Scheme 3). 有效地提升 了膦氧化物 $\mathbf{P} 2 \cdot[\mathbf{O}]$ 的还原速率, 将反应温度由原来的 $100{ }^{\circ} \mathrm{C}$ 降低到室温, 并进一步拓宽了底物适用范围, 但 收率和 $Z / E$ 值没有得到提升.

随后，该课题组 ${ }^{[14]}$ 进一步对膦杂环氧化物催化剂 前体进行设计和篮选, 发现 P3・[O] 可以使得非稳定的膦 叶立德也适用于此氧化还原催化循环体系完成 Wittig 反 应(Scheme 4).

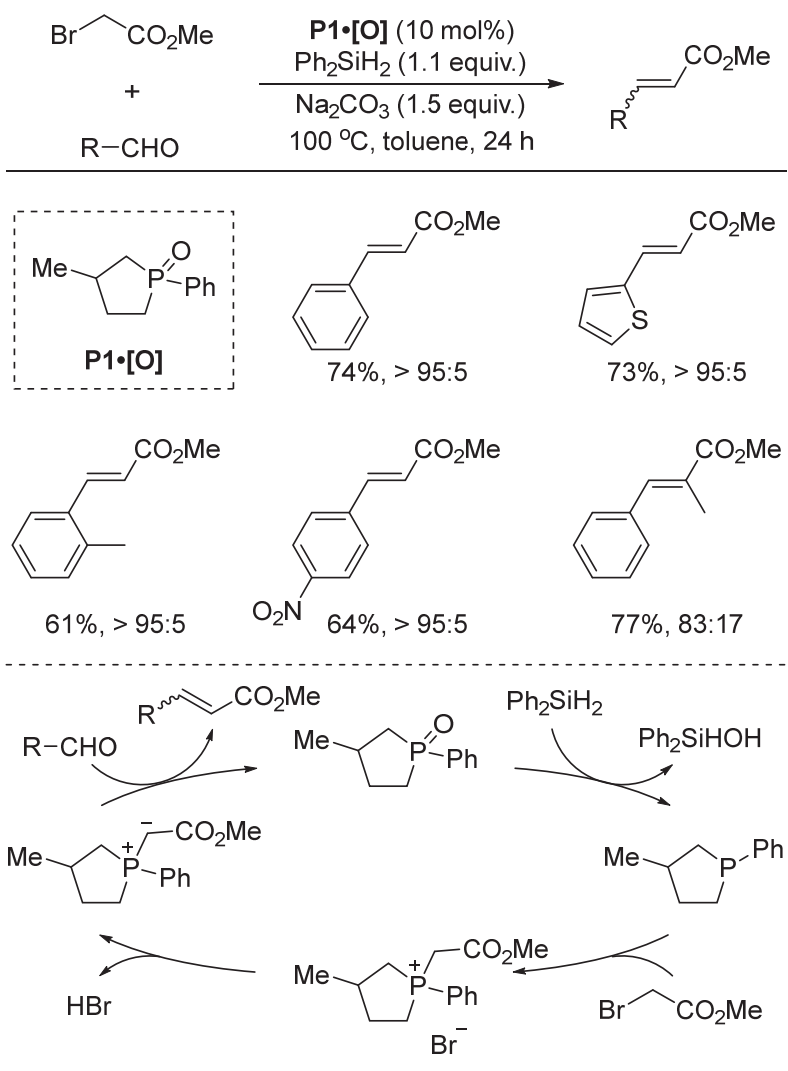

图式 2 稳定的叶立德参与的催化 Wittig 反应

Scheme 2 Catalytic Wittig reaction with stabilized ylides

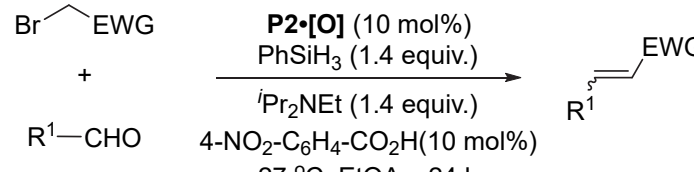

$$
\begin{aligned}
& 27^{\circ} \mathrm{C} \text {, EtOAc, } 24 \mathrm{~h}
\end{aligned}
$$

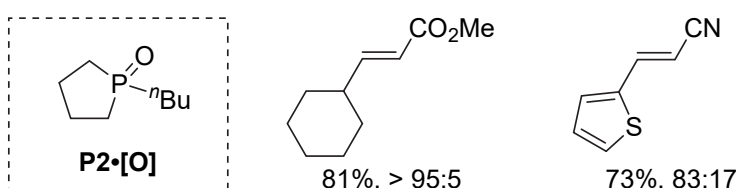

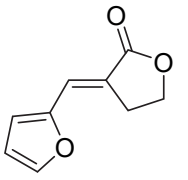

$61 \%, 90: 10$
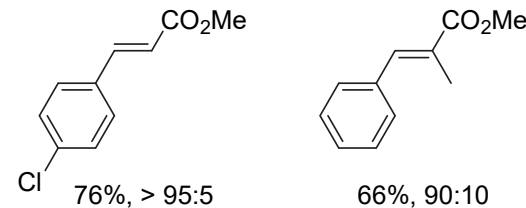

$66 \%, 90: 10$
图式 3 室温下的催化 Wittig 反应

Scheme 3 Catalytic Wittig reaction in room temperature

2010 年, 周剑课题组 ${ }^{[15]}$ 发展了废物再利用策略, 改 善 Wittig 反应效率，通过串联反应的方法，在完成第一 步的 Wittig 反应后，把生成的 $\mathrm{R}_{3} \mathrm{P}=\mathrm{O}$ 副产物作为催化 剂或者共催化剂, 实现废物的再利用(Scheme 5). 在硅 烷的作用下，也可以实现第二步反应的催化过程. 


\begin{tabular}{|c|c|c|}
\hline $\mathrm{Br} \widehat{\mathrm{R}^{2}}$ & $\begin{array}{c}\mathrm{P} 3 \cdot[\mathrm{O}](10 \text { mol\%) } \\
\mathrm{Ph}_{2} \mathrm{SiH}_{2}(1.2 \text { equiv. })\end{array}$ & $R^{2}$ \\
\hline $\mathrm{R}^{1}-\mathrm{CHO}$ & $\begin{array}{l}{ }^{i} \mathrm{Pr}_{2} \mathrm{NEt} \text { (1.2 equiv.) } \\
140^{\circ} \mathrm{C} \text {, toluene, } 24 \mathrm{~h}\end{array}$ & $\mathrm{R}^{1}$ \\
\hline
\end{tabular}

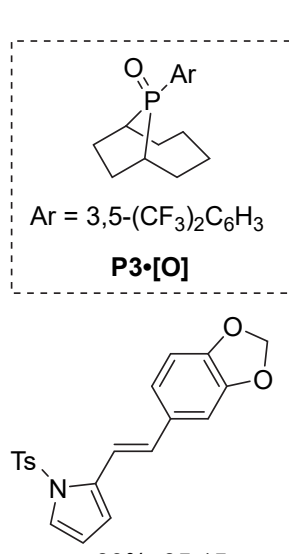

$89 \%, 85: 15$
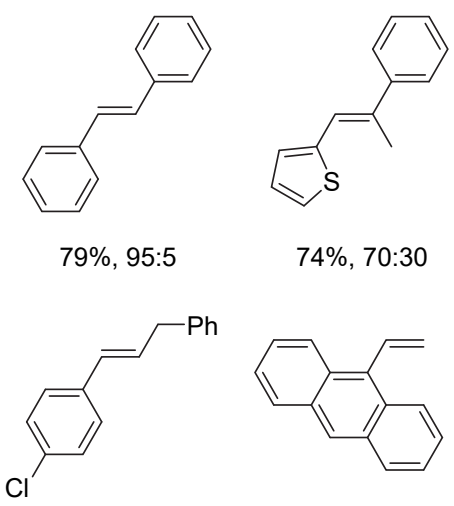

$74 \%, 75: 25$

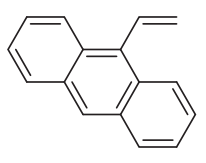

$79 \%, 95: 5$

图式 4 非稳定的叶立德参与的催化 Wittig 反应 Scheme 4 Catalytic Wittig reaction with unstabilized ylides

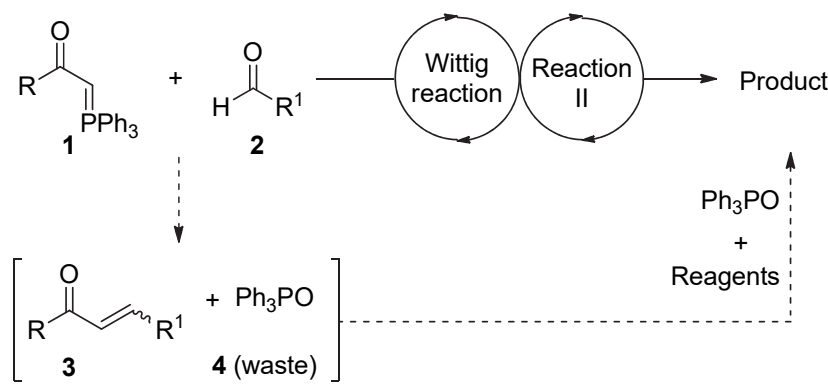

图式 5 串联 Wittig 反应/催化还原反应

Scheme 5 A tandem Wittig/conjugate-reduction reaction.

2019 年, Werner 课题组 ${ }^{[16]}$ 发展了四元膦杂环氧化物 $\mathbf{P 4} \cdot[\mathbf{O}]^{[17]}$ 作为催化剂前体, 在氧化还原催化循环的体系 中可以以 $1 \mathrm{~mol} \%$ 的催化剂用量, 在不需要布朗斯特酸 添加剂, 室温条件下以很好地收率和 $Z / E$ 选择性完成 Wittig 反应(Scheme 6). 简单的膦氧化物(比如 $\mathrm{Ph}_{3} \mathrm{PO}$, $\mathrm{Bu}_{3} \mathrm{PO}$ 等)也被尝试应用于催化 Wittig 反应当中, 但是往 往需要高温、微波加热等苛刻的反应条件 ${ }^{[18-19]}$.

2014 年, Werner 课题组 ${ }^{[20-21]}$ 报道了首例通过 P(III)/ $\mathrm{P}(\mathrm{V})$ 氧化还原循环过程实现不对称催化 Wittig 反应 (Scheme 7). 作者通过对大量手性三价膦催化剂的篎选, 最终确定使用 $(S, S)-\mathrm{Me}-\mathrm{DuPhos}$ (P5)作为催化剂, 苯基 硅烷作为还原剂, 微波加热的条件下可以实现前手性卤 代酮的去对称环化, 以 $62 \%$ 的 $e e$ 值非对映选择性的得 到二酮类产物.

三价膦催化剂可以在无布朗斯特碱的条件下通过 对缺电子烯烃的亲核进攻/质子迁移得到膦叶立德中间 体. 基于这一理论, Werner 和林文伟课题组 ${ }^{[22-25]}$ 发展

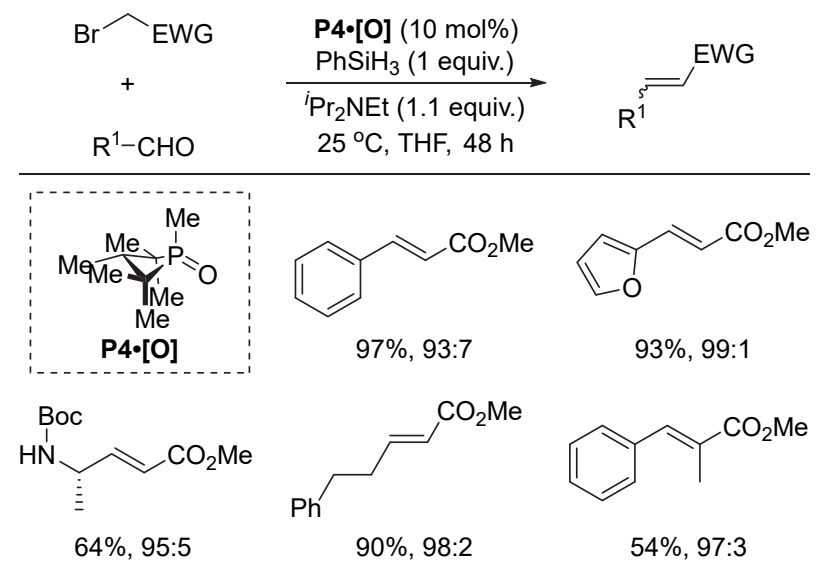

图式 6 高张力催化剂前体参与的催化 Wittig 反应 Scheme 6 Catalytic Wittig reaction with high tension precatalyst

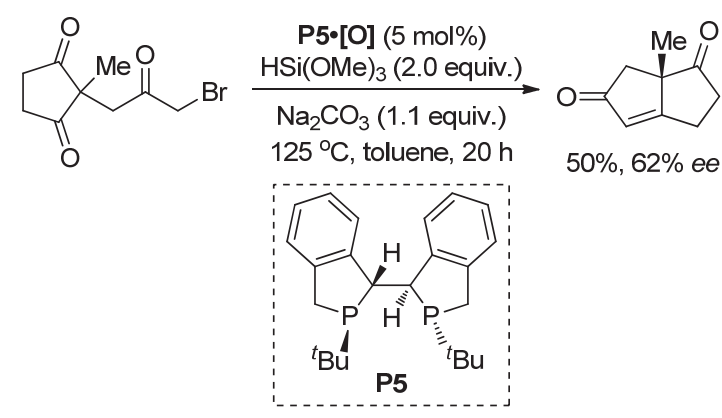

图式 7 双膦催化剂参与的催化 Wittig 反应

Scheme 7 Catalytic Wittig reaction with bisphosphine catalyst

了 $\mathrm{P}(\mathrm{III}) / \mathrm{P}(\mathrm{V})$ 氧化还原循环过程催化丁烯二酸二乙酯与 芳醛的 Wittig 反应(Scheme 8A). 这一催化模式也被应 用到更多的底物中: Vouturiez 课题组 ${ }^{[26-27]}$ 发展了炔酸酯 与醛基吡咯的加成串联分子内 Wittig 反应来构筑氮杂并 环(Scheme 8B); Kwon 课题组 ${ }^{[28]}$ 发展了联烯酸酯与邻氨 基苯甲醛的 $\gamma$-加成串联分子内 Wittig 反应来构筑二氢喹 啉(Scheme 8C); 黄有课题组 ${ }^{[29]}$ 发展了 Morita-BaylisHillman (MBH)碳酸酯与邻氨基查耳酮的烯丙基烷基化 串联分子内 Wittig 反应来构筑 6-6-5 并环 (Scheme 8D).

2018 年, Vouturiez 课题组 ${ }^{[30]}$ 基于这一反应模式, 发 展了其不对称催化版本(Scheme 9). 在 Kwon 发展的桥 环催化剂 P8-[O]条件下, 丁炔二酸二酯与 1,3-二羰基化 合物经历极性反转加成/分子内 Wittig 反应得到一系列 三氟甲基取代的环丁烯化合物. 反应有中等到很好的收 率和优秀的对映选择性.

\section{2 催化 Staudinger 反应和氮杂 Wittig 反应}

2012 年, van Delft 和 Rutjes ${ }^{[31]}$ 报道了首例经过 $\mathrm{P}(\mathrm{III}) / \mathrm{P}(\mathrm{V})$ 氧化还原循环过程的催化 Staudinger 反应 (Scheme 10). 作者使用二苯并膦杂环戊二烯 P9 作为催 


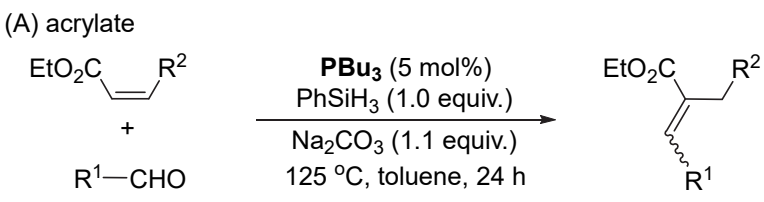

(B) ynoate

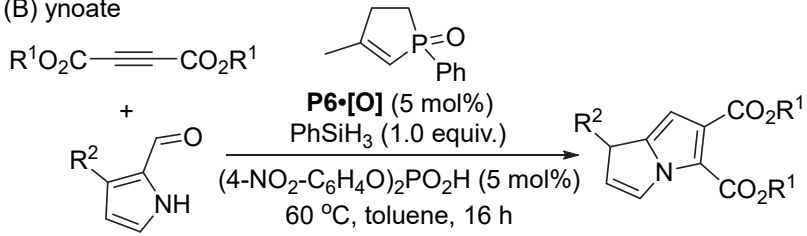

(C) allene<smiles></smiles>
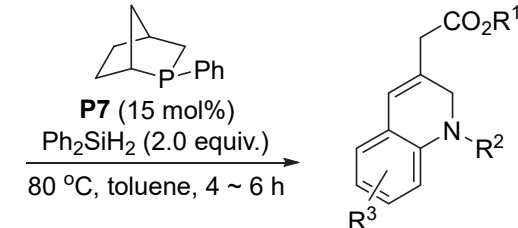

(D) $\mathrm{MBH}$ carbonates

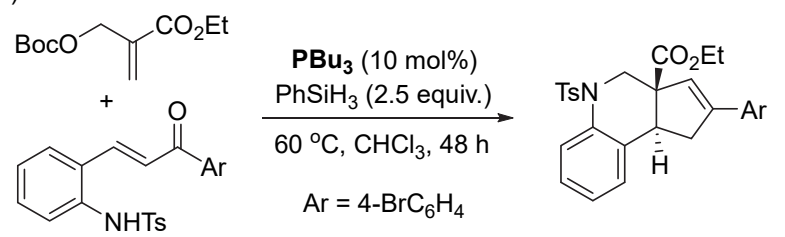

图式 8 其他类型的叶立德前体及催化 Wittig 反应模式 Scheme 8 Other ylide precursors and catalytic Wittig reaction modes

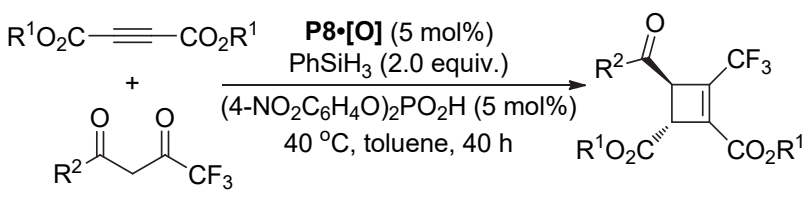

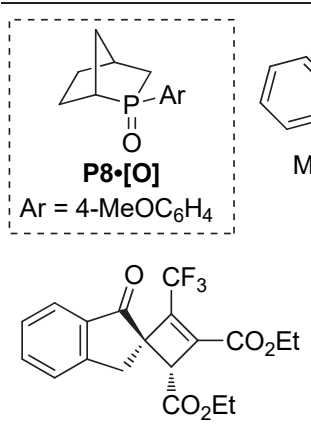

$53 \%, 90 \%$ ee

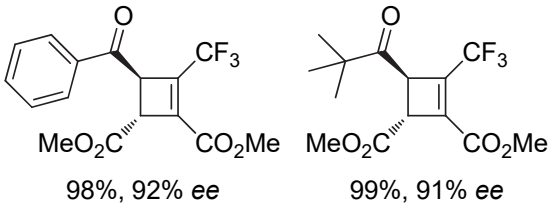

图式 9 不对称催化 Wittig 反应

Scheme 9 Asymmetric catalytic Wittig reaction

化剂, $\mathrm{PhSiH}_{3}$ 作为还原剂, 在回流的条件下将叠氮化合 物转化为胺类. 反应有很好的官能团耐受性和较好的收 率. 同时, 作者通过对于 ${ }^{31} \mathrm{P}$ NMR 的监测, 初步确认了 氧化还原循环催化的机理. 不同于经典的剂量膦试剂参 与 Staudinger 反应里的亚胺基膦水解的过程, 此反应中 亚胺基膦通过硅烷还原可以直接得到目标产物并释放 出催化剂 P9, 完成催化循环.

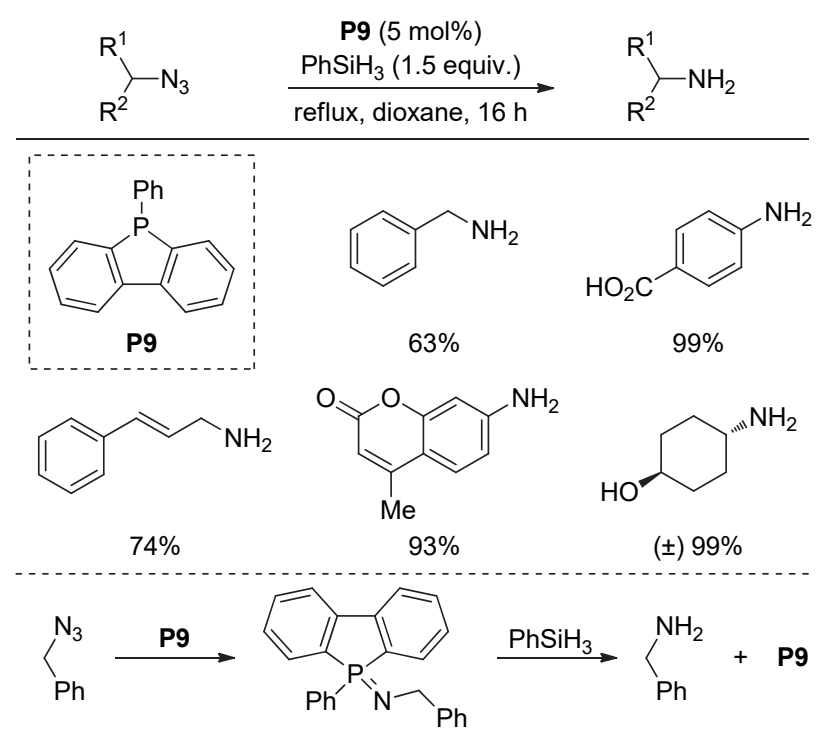

图式 10 催化 Staudinger 反应

Scheme 10 Catalytic Staudinger reaction

简单的三苯基膦也可以完成催化 Staudinger 反应 (Scheme 11). Mecinović ${ }^{[32]}$ 证实在 $\left(\mathrm{PhSiH}_{2}\right)_{2} \mathrm{O}$ 作为还原剂 的条件下，尽管反应时间有所延长，但是反应有很好的 收率和官能团耐受性. 最重要的是反应可以在室温下进 行.

$$
\begin{aligned}
& \text { (1) } \mathrm{PPh}_{3} \text { (10 mol\%) } \\
& \left(\mathrm{PhSiH}_{2}\right)_{2} \mathrm{O} \text { (1.5 equiv.) } \\
& \mathrm{R}-\mathrm{N}_{3} \quad \stackrel{\text { r.t., } \mathrm{CPME}, 24 \mathrm{~h}}{\text { (2) } \mathrm{H}_{2} \mathrm{O}(10 \text { equiv. })} \mathrm{R}-\mathrm{NH}_{2} \cdot \mathrm{HCl}
\end{aligned}
$$$$
\text { (3) } 4.0 \mathrm{~mol} / \mathrm{L} \mathrm{HCl} \text { in dioxane }
$$<smiles>ClCCNCc1cncs1</smiles>

图式 $11 \mathrm{PPh}_{3}$ 介导的催化 Staudinger 反应

Scheme $11 \mathrm{PPh}_{3}$-mediated catalytic Staudinger reaction

$\mathrm{P}(\mathrm{III}) / \mathrm{P}(\mathrm{V})$ 氧化还原循环过程的催化 Staudinger 反 应在 2012 年被 Ashfeld 课题组 ${ }^{[33]}$ 首次应用到酰胺化反应 中，避免了分离伯胺的步骤，以及处理计量膦氧化物的 副产物(Scheme 12). 该反应底物范围广，分子间和分子 内的酰胺化均适用, 对于含裸露氨基的底物也耐受. 作 者认为机理如下: 三苯基膦与叠氮化合物反应得到亚胺 基膦中间体，进而与羧酸发生亲核加成，接着通过分子 内重排实现酰胺化并释放出三苯基氧膦，并在硅烷的还 原下得到三苯基膦，完成氧化还原循环催化. 后续有文 献提出了其他可能的机理 ${ }^{[34-35]}$, 目前对于此反应的机理 尚无定论

2013 年, van Delft 和 Rutjes ${ }^{[36]}$ 报道了通过 P(III)/ $P(V)$ 氧化还原循环过程得到亚胺基膦中间体参与的催 化 Staudinger-氮杂 Wittig 反应(Scheme 13). 通过对底物 


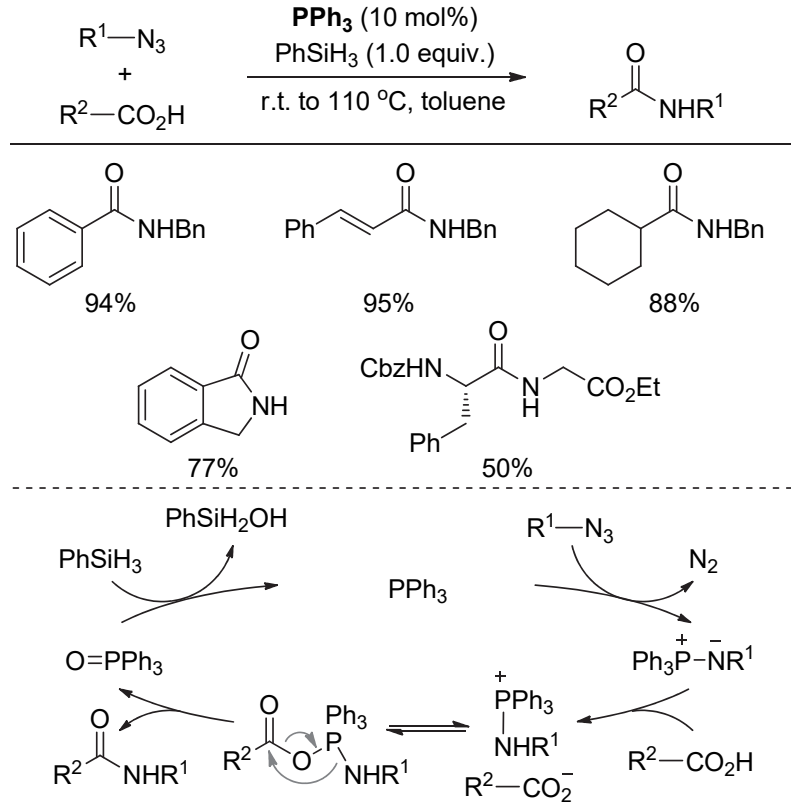

图式 12 催化 Staudinger 酰胺化反应

Scheme 12 Catalytic Staudinger amidation reaction

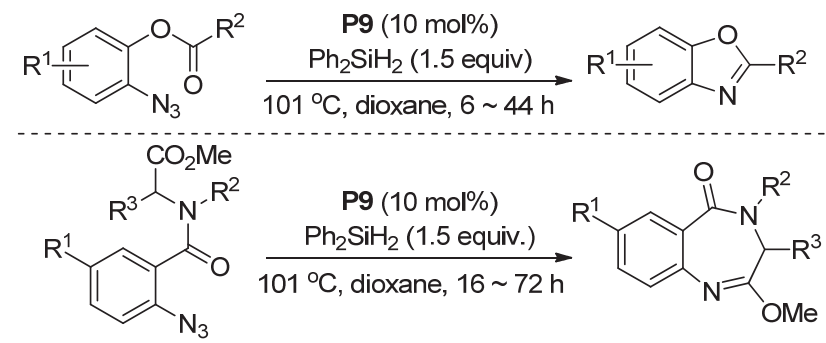

图式 13 催化 Staudinger-氮杂 Wittig 反应

Scheme 13 Catalytic Staudinger- $A z a$-Wittig reaction

的设计, 可以中等的收率得到苯并氮杂五元环和七元环 化合物。

2014 年，丁明武课题组 ${ }^{[37]}$ 使用相同的策略，报道了 简单的三苯基膦作为催化剂, 在空气中稳定存在的四甲 基二硅氮烷(TMDS)作为还原剂的条件下，高效构筑一 系列喹唑啉酮类化合物的策略(Scheme 14). 反应中添 加的四异丙氧基钛有助于膦氧化合物的还原.

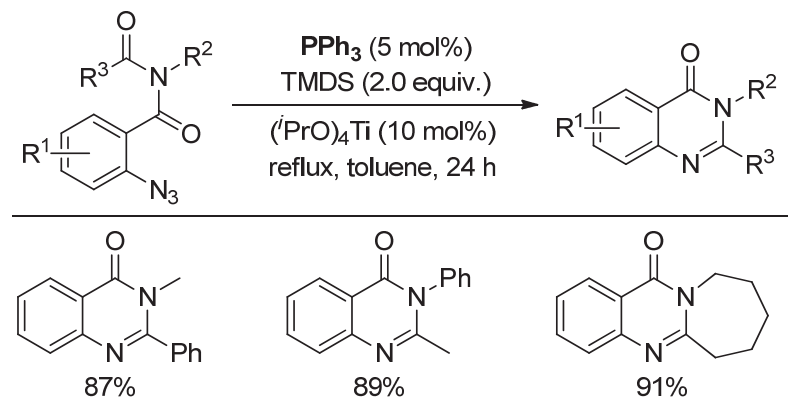

图式 $14 \mathrm{PPh}_{3}$ 介导的催化 Staudinger-氮杂 Wittig 反应 Scheme $14 \mathrm{PPh}_{3}$-mediated catalytic Staudinger- $A z a$-Wittig reaction
接着作者 ${ }^{[38]}$ 发现在类似的条件下可以实现叠氮羧 酸与酰氯一锅/分步的分子内催化 Staudinger-氮杂 Wittig 反应(Scheme 15). 通过对底物的设计，可以得到氮杂五 元环或六元环产物. 值得指出的是, 作者证明反应中添 加的 $\mathrm{Cu}(\mathrm{OTf})_{2}$ 是三苯基氧膦还原为三苯基膦的关键.

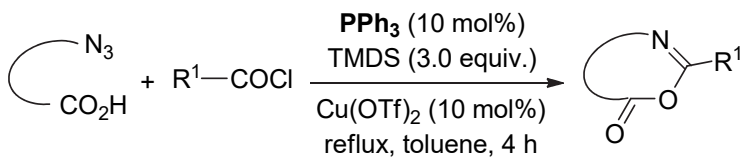

图式 15 一锅法催化 Staudinger-氮杂 Wittig 反应 Scheme 15 One-pot catalytic Staudinger- $A z a$-Wittig reaction

2014 年, Herdewijn 课题组 ${ }^{[39}$ 报道了首例有机膦催 化重氮化合物的氮杂 Wittig 反应(Scheme 16). 在仲醇被 完全氧化为酮后，通过简单的过滤即可将其应用于 $\mathrm{P}(\mathrm{III}) / \mathrm{P}(\mathrm{V})$ 氧化还原循环催化过程，接着经历分子内环 化反应来构筑二氮杂苯酚类化合物.<smiles>[R]C(O)C([R])C(=O)C(=[W])C#N</smiles><smiles>[R]c1nnc(C(C)(C)C)c(O)c1[R]</smiles>

图式 16 重氮化合物的催化氮杂 Wittig 反应 Scheme 16 Catalytic aza-Wittig reaction of diazonium compounds

2006 年, Marsden 课题组 ${ }^{[40-41]}$ 发展了首例不对称氮 杂 Wittig 反应，然而反应中用到了计量的手性膦催化剂. 2018 年, Kwon 课题组 ${ }^{[42]}$ 通过 $\mathrm{P}(\mathrm{III}) / \mathrm{P}(\mathrm{V})$ 氧化还原循环 过程完成了其催化不对称版本(Scheme 17). 反应在布 朗斯特酸的协助下进行，经过对二酮化合物的去对称 化，以优秀的收率和对映选择性得到含亚胺官能团的杂 环化合物.

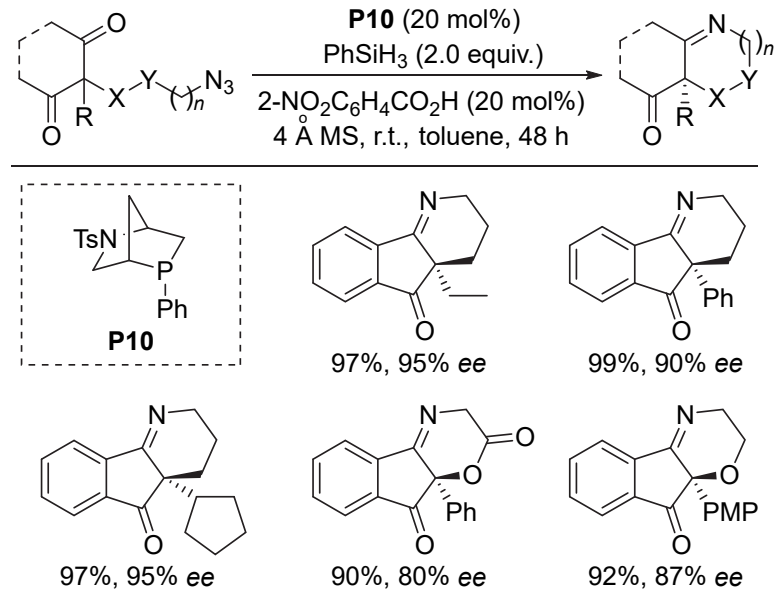

图式 17 催化不对称氮杂 Wittig 反应

Scheme 17 Catalytic enantioselective aza-Wittig reaction 


\section{3 催化 Appel 反应}

2011 年, van Delft 和 Rutjes ${ }^{[43]}$ 实现了首例 P(III)/P(V) 氧化还原循环的催化 Appel 溴化反应(Scheme 18). Appel 反应作为经典的有机膦介导的缩合反应, 往往面临试剂 兼容性的问题(卤代物和硅烷). 而在本反应中, 溴丙二 酸二乙酯 $(\mathrm{DEBM})$ 是一种理想的溴供体, 与氢硅烷还原 剂具有良好的相容性, 使得反应有较好的官能团耐受 性. 作者从 ${ }^{31} \mathrm{P}$ NMR 角度出发初步判断了此反应的机 理: 催化剂 P9 仅能和溴源发生反应得到季鏻盐, 不会 和产物中的溴代烷基发生反应, 有很好的化学选择性. 季鏻盐与醇羟基结合后通过 $\mathrm{S}_{\mathrm{N}} 2$ 反应得到目标产物, 释 放出膦氧化物, 在硅烷的还原下得到 $\mathbf{P 9}$ 完成催化循环.

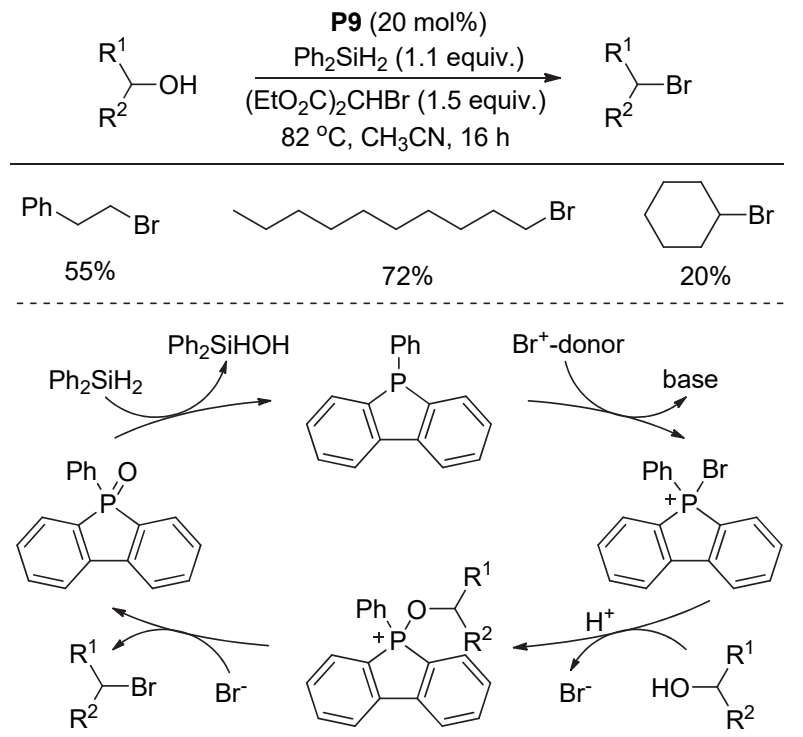

图式 18 催化 Appel 溴化反应

Scheme 18 Catalytic Appel bromination reaction

Werner 课题组 ${ }^{[44]}$ 于 2019 年将三氯甲苯作为氯源, 三辛基膦作为催化剂, 实现了基于 $\mathrm{P}(\mathrm{III}) / \mathrm{P}(\mathrm{V})$ 氧化还原 循环的催化 Appel氯代反应(Scheme 19). 反应无需溶剂, 有很好的产率和官能团耐受性.

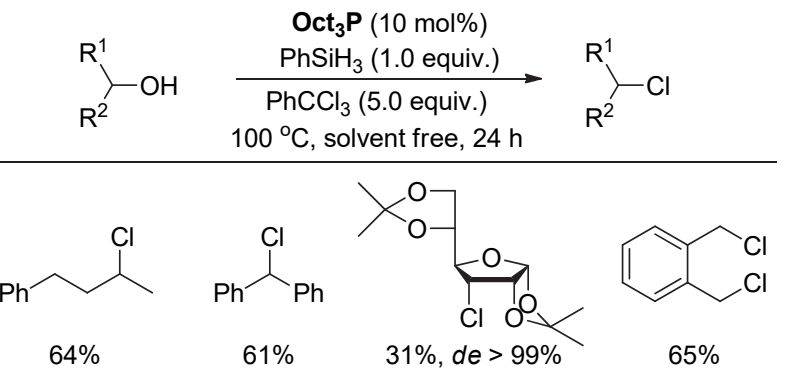

图式 19 催化 Appel 氯代反应

Scheme 19 Catalytic Appel chlorination reaction

2014 年, Mecinović 课题组 ${ }^{[45]}$ 在三苯基膦作为催化 剂, 适量布朗斯特酸作为添加剂的条件下, 通过 P(III)/
$\mathrm{P}(\mathrm{V})$ 氧化还原循环实现了 Appel 酰胺化反应(Scheme 20). 反应有很好的官能团耐受性，但收率较低. 作者推 测反应经过如下的机理: 三苯基膦与四氯甲烷反应生成 季鏻盐后, 通过与羧酸负离子的 $\mathrm{S}_{\mathrm{N}} 2$ 作用成为与羧酸负 离子结合的季鏻盐. 这一活泼物种与胺类化合物发生酰 胺化后脱除三苯基氧膦. 紧接着在硅烷的还原下完成催 化循环。

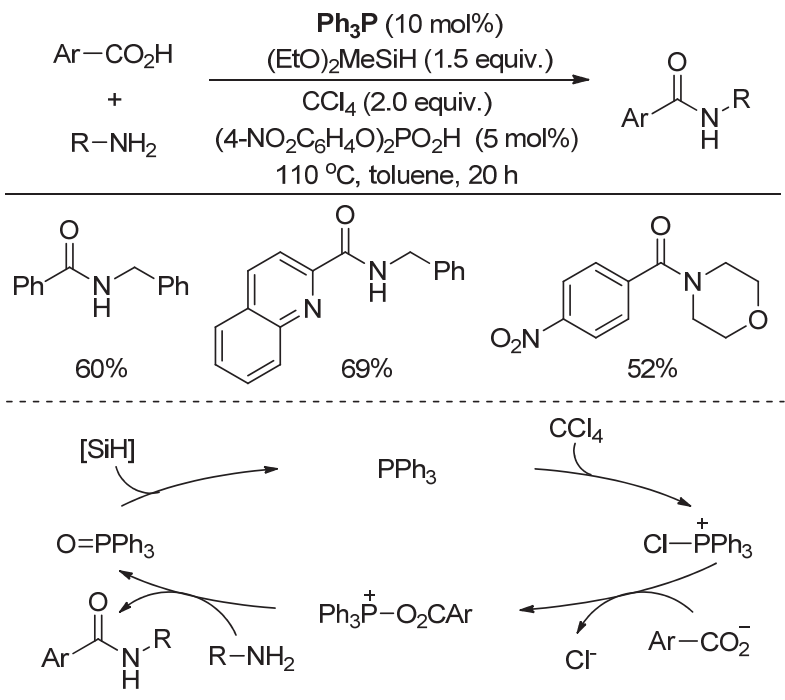

图式 20 催化 Appel 酰胺化反应

Scheme 20 Catalytic Appel amidation reaction

2019 年, Radosevich 课题组 ${ }^{[46]}$ 使用 P4•[O]作为催化 剂, 苯基硅烷作为还原剂, 实现了胺和羧酸的串联环化 反应从而高效构筑氮杂环 (Scheme 21). 催化剂只和 DEBE 反应而不与底物中的溴原子反应，含羧酸官能团 的药物分子参与环化反应时，相邻的手性中心几乎不受 影响，产物可以是 6-6-5 并环，也可以是 6-7-5 并环. 作 者认为反应中先生成季鏻盐促进酰胺化反应，接着通过 经典的 Bischler-Napieralski 反应完成环化, 并释放出三 苯基氧膦，最后在硅烷的还原下实现催化循环。

\section{4 催化 Mitsunobu 反应}

Mitsunobu 反应和 Appel 反应都是由膦介导的缩合 反应. 2010 年, $\mathrm{O}^{\prime}$ Brien 课题组 ${ }^{[47]}$ 基于 $\mathrm{P}(\mathrm{III}) / \mathrm{P}(\mathrm{V})$ 氧化还 原循环的思路, 实现了催化 Mitsunobu 反应(Scheme 22). 从动力学的角度来讲, 醇羟基的 $\mathrm{C}-\mathrm{O}$ 键键能较强, 因 此反应在此依旧使用了剂量的偶氮二甲酸二异丙酯 (DIAD)来活化醇羟基.

随后, Aldrich 课题组 ${ }^{[48]}$ 通过同时使用硅烷还原膦氧 化物, 铁催化氧化实现肼的循环, 实现了 Mitsunobu 反 应完全的催化(Scheme 23). 不过操作繁琐，反应耐受性 差仍是需要解决的问题. 而且有文献指出反应不一定完 全是催化的过程 ${ }^{[49-50]}$. 当反应被应用到对映选择性产物 
<smiles>Nc1cc[R1]cc1-n1cccc1</smiles>

P4·[O] (15 mol\%) $\underset{\mathrm{DEBE}(2.4 \text { equiv. })}{\stackrel{\mathrm{PhSiH}_{3} \text { (2.2 equiv.) }}{\longrightarrow}}$ $80^{\circ} \mathrm{C}, \mathrm{DCE}, 12 \sim 14 \mathrm{~h}$

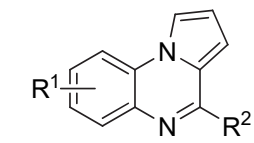

$\mathrm{R}^{2}-\mathrm{CO}_{2} \mathrm{H}$<smiles>CCCc1nc2ccccc2n2cccc12</smiles>
$90 \%$<smiles></smiles><smiles></smiles><smiles>COc1ccc2cc([C@@H](C)c3nc4ccccc4n4cccc34)ccc2c1</smiles>

$68 \%, 85 \%$ ee

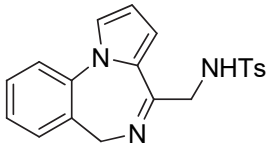

$66 \%$

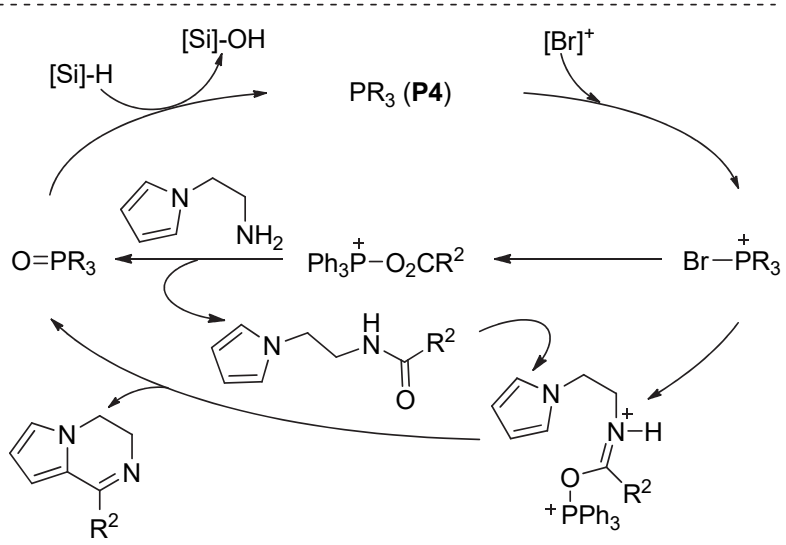

图式 21 膦介导的串联催化环化反应

Scheme 21 Phosphetane-mediated tandem annulation reaction

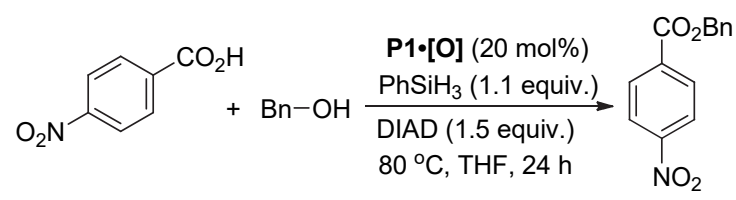

图式 22 催化 Mitsunobu 反应

Scheme 22 Catalytic Mitsunobu reaction

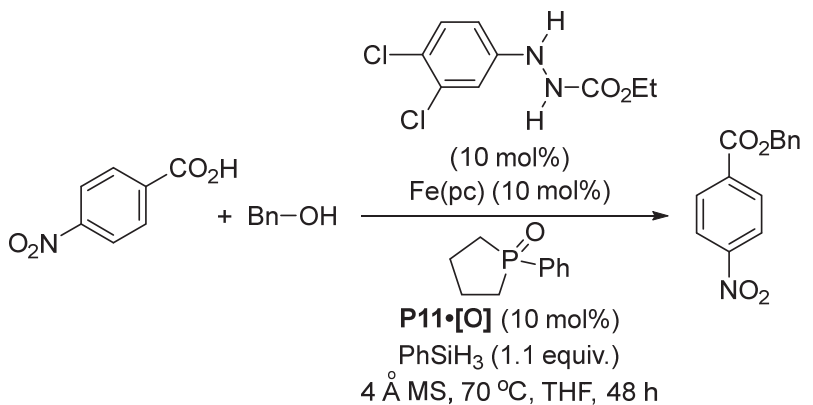

图式 23 膦氧化物和偶氮二甲酸二酯双催化循环

Scheme 23 Recycling both phosphine oxide and the azocarboxylate reagent

合成中时没有表现出 Mitsunobu 反应该有的立体选择 性, 并且在无需肼催化剂的条件下，反应也可以发生.
2019 年, Denton 课题组 ${ }^{[51]}$ 通过催化剂的设计，完美 地实现了催化 Mitsunobu 反应(Scheme 24). 反应无需肼 的参与, 绕开了氧化还原循环的过程, 避免了剂量膦氧 化合物的产生，有很好的收率、官能团耐受性和立体选 择性.
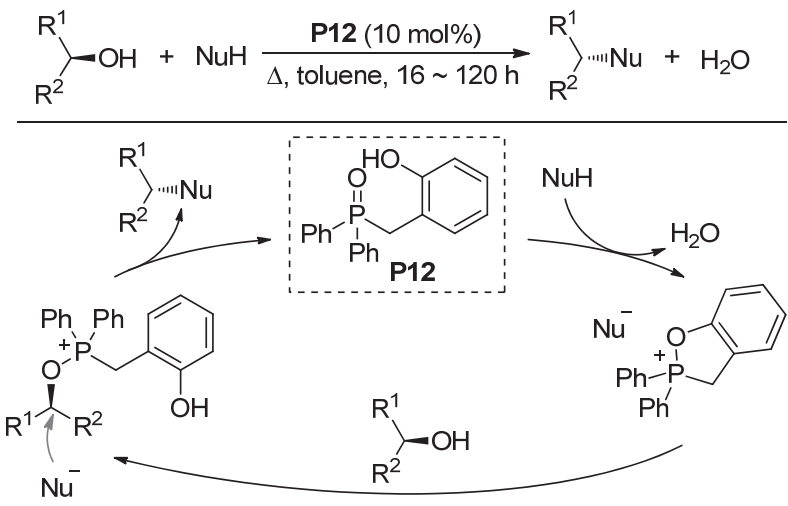

图式 24 氧化还原中性的催化 Mitsunobu 反应

Scheme 24 Redox-neutral catalytic Mitsunobu reaction

\section{5 催化还原氧原子迁移}

由于 $\mathrm{P}(\mathrm{III})$ 有很好的还原性，因此有机膦化合物是 各种含氧底物出色的氧原子受体. 2010 年, Woerpel 课题 组 ${ }^{[52]}$ 实现了首例通过 $\mathrm{P}(\mathrm{III}) / \mathrm{P}(\mathrm{V})$ 氧化还原循环的氧原子 迁移策略，将烷基过氧硅醚选择性还原为烷基硅醚 (Scheme 25). 反应有较好的官能团耐受性和中等偏上 的收率. 作者通过标记实验证明，反应由三苯基膦插入 过氧键启动，经过协同消除/硅基迁移得到目标产物. 过 程中生成的膦氧化物由原位生成的氢化钛还原, 完成催 化循环.

$$
\left.\underset{\mathrm{R}^{2}}{\mathrm{R}^{1}}\right\rangle-\mathrm{OH}+\mathrm{NuH} \frac{\mathrm{P} 12(10 \mathrm{~mol} \%)}{\Delta \text {, toluene, } 16 \sim 120 \mathrm{~h}} \underset{\mathrm{R}^{2}}{\mathrm{R}^{1} \cdot \cdots+\mathrm{Nu}}+\mathrm{H}_{2} \mathrm{O}
$$

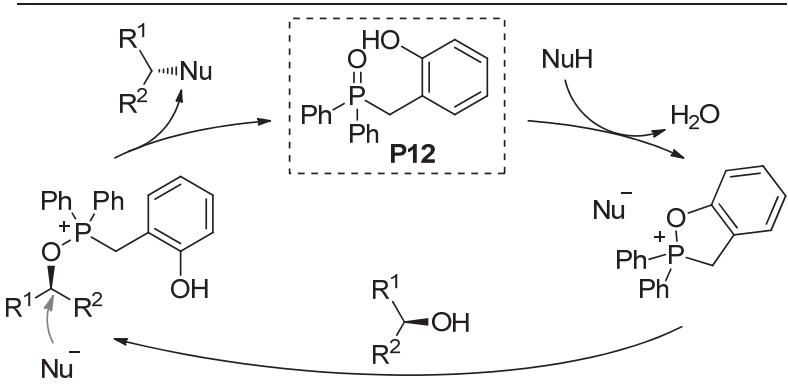

图式 25 催化还原氧原子迁移

Scheme 25 Catalytic reductive O-atom transfer

Radosevich 课题组 ${ }^{[53]}$ 在 2015 年将这一氧原子迁移 的策略应用到 $\alpha$-酮酸酯与羧酸的脱氧偶联中 (Scheme 26). 在 P13•[O]作为催化剂时, 反应有很好的官能团耐 受性，甚至底物中含有芳胺官能团的反应也有较好的收 
率. 作者认为反应由 Kukhtin-Ramirez 加成 ${ }^{[54]}$ 启动，接 着经过质子迁移 $\mathrm{S}_{\mathrm{N}} 2$ 反应得到目标产物, 并释放出膦氧 化物, 通过苯基硅烷的还原完成催化循环.

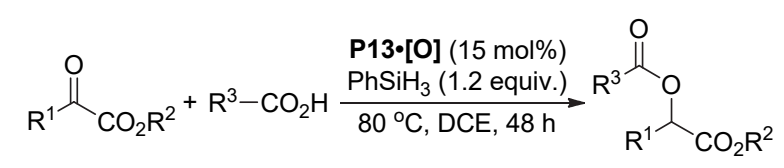
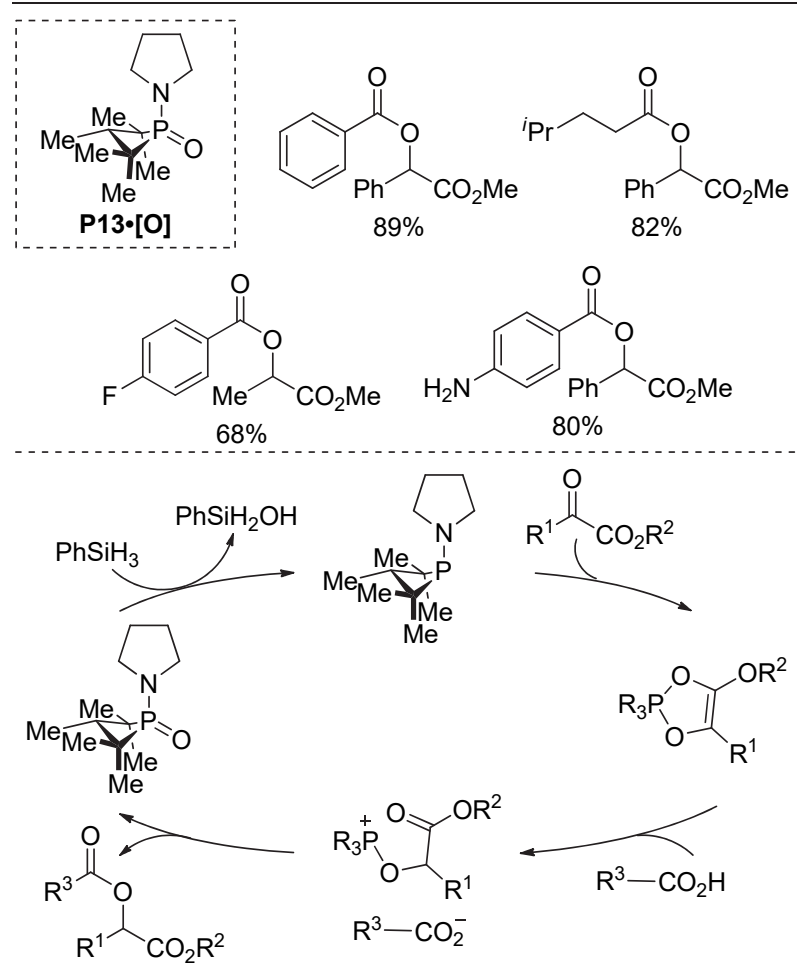

图式 26 膦介导的催化脱氧缩合反应

Scheme 26 Phosphetane-mediated catalytic deoxygenative condensation reaction

有机膦和硝基反应也可以起到氧原子迁移的作用. Cadogan 课题组 ${ }^{[55-57]}$ 在这一方面做了开创性的工作, 然 而所用的有机膦都是剂量的. 2017 年, Radosevich 课题 组 $\left.{ }^{58}\right]$ 使用 $\mathbf{P 4} \cdot[\mathrm{O}]$ 作为催化剂, 通过 $\mathrm{P}(\mathrm{III}) / \mathrm{P}(\mathrm{V})$ 氧化还原 循环的策略实现了硝基与偶氮分子内反应来催化合成 吲唑和苯并三唑化合物, 有中等到很好的产率和很好的 官能团耐受性(Scheme 27). 硝基经过两次有机膦化合 物 P4 对其进行氧原子迁移, 接着氮原子进攻关环得到 目标产物, 释放出 $\mathbf{P 4} \cdot[\mathbf{O}]$, 最后在苯基硅烷的还原下完 成催化循环。此外，作者还通过密度泛函理论(density functional theory, DFT)计算, 说明该反应的决速步为硝 基和有机膦的 $[3+1]$ 环加成. 并且证实了高张力的环烷 基膦相对于没有环状结构的三烷基膦更适用于此类反 应. 相比来讲, Nazare 课题组 ${ }^{[59]}$ 使用了环系更大的膦杂 五元环催化剂 P6・[0]也完成了相同的反应, 但所需的催 化剂用量大大提高, 反应所需时间也有所延长.

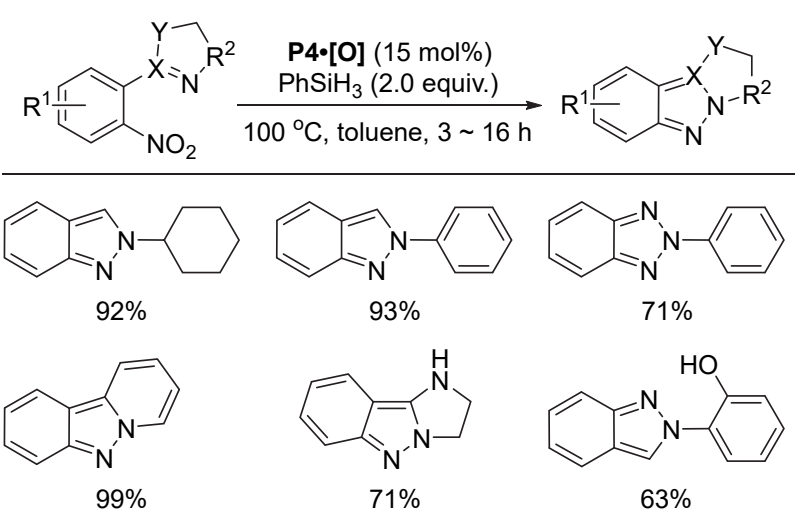

图式 27 膦介导的 Cadogen 杂环化反应

Scheme 27 Phosphetane-mediated Cadogan heterocyclization reaction

随后, Radosevich 课题组 ${ }^{[60] 又}$ 将这一硝基脱氧的反 应模式应用到吲哚和咔唑的合成中(Scheme 28). 使用 相同的催化剂前体 P4・[O]，作者在低温下捕捉到了第二 次氧原子迁移的关键中间体，并进一步通过 DFT 计算 证实在高温条件下，该中间体可以通过裂解变为催化剂 前体和游离的胺，最后通过分子内 $\mathrm{C}-\mathrm{H}$ 胺化得到吲哚 或咔唑类化合物 ${ }^{[1]]}$.

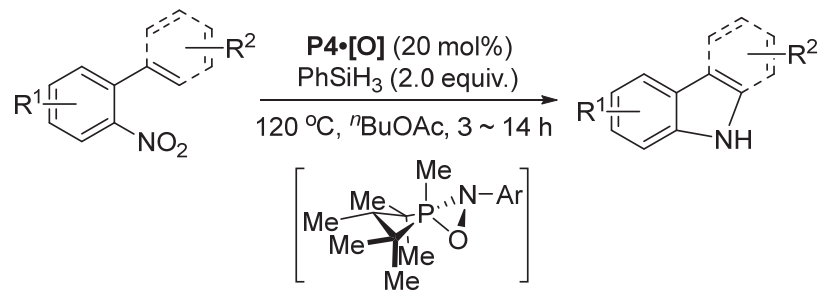

图式 28 膦介导的 $\mathrm{C}-\mathrm{H}$ 胺化环化反应

Scheme 28 Phosphetane-mediated $\mathrm{C}-\mathrm{H}$ amination/cyclization reaction

鉴于这一中间体可以在路易斯酸的作用下开环, Radosevich 课题组 ${ }^{[2]}$ 将这一催化体系延伸到与硼酸化 合物的偶联中, 并成功实现了基于 $\mathrm{P}(\mathrm{III}) / \mathrm{P}(\mathrm{V})$ 氧化还原 循环的催化 $\mathrm{C}-\mathrm{N}$ 键偶联反应，得到了一系列芳胺类化 合物(Scheme 29). 反应的官能团耐受性好, 存在裸露时 反应也可以顺利进行，含有手性氨基酸取代基时反应对 氨基酸骨架也不造成影响. 重要的是廉价易得的硝基甲 烷可以作为胺源实现芳胺的合成, 有很好的应用价值.

2020 年, Radosevich 课题组 ${ }^{[63]}$ 发现这一芳胺化的策 略还可以进一步延伸到串联环化反应中. 通过对底物的 设计，芳胺化得到的氨基可以进一步发生分子内酰胺化 或者缩合反应得到四种骨架的高度官能团化的苯并氮 杂环化合物(Scheme 30). 


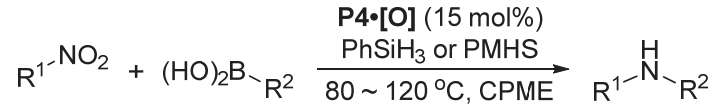

$$
\begin{aligned}
& 3 \sim 14 \mathrm{~h}
\end{aligned}
$$<smiles>Ic1cccc(Nc2ccccc2)c1</smiles>

$86 \%$<smiles>CNc1ccc(Br)cc1</smiles><smiles>CNc1cncnc1</smiles><smiles>CNc1cccc(CC(NC(=O)OC(C)(C)C)C(C)(C)C)c1</smiles>

图式 29 膦介导的 $\mathrm{C}-\mathrm{N}$ 还原偶联

Scheme 29 Phosphetane-mediated reductive $\mathrm{C}-\mathrm{N}$ cross-coupling

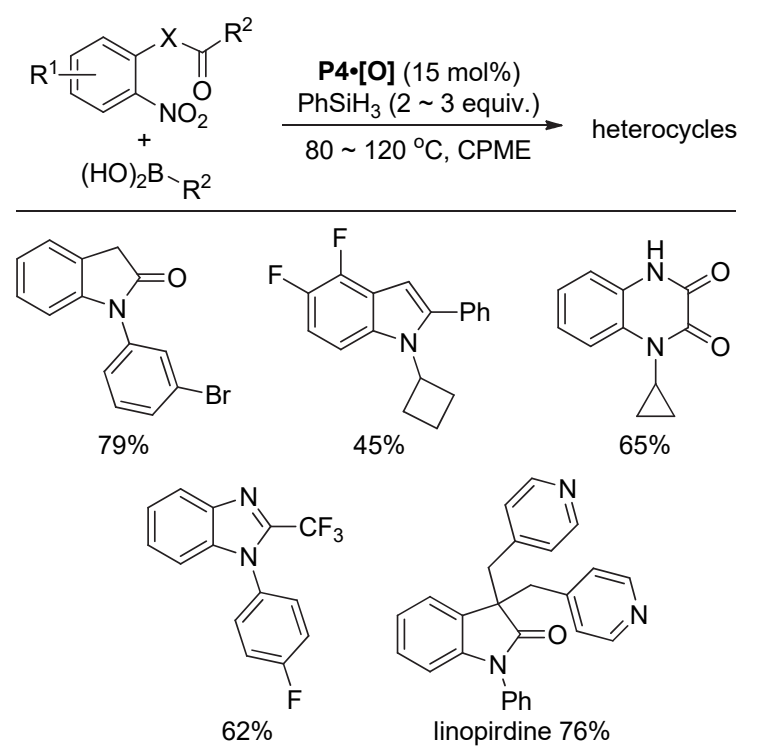

图式 30 膦介导的串联反应

Scheme 30 Phosphetane-mediated tandem reaction

有机膦催化剂除了可以对羰基、硝基等官能团进行 氧原子迁移, Radosevich 课题组 ${ }^{[64]}$ 还在 2019 年报道了对 于磺基的基于 $\mathrm{P}(\mathrm{III}) / \mathrm{P}(\mathrm{V})$ 氧化还原循环的催化氧原子迁 移过程, 实现了吲哚 3-位的颈基化反应(Scheme 31). 反 应有很好的官能团耐受性，硝基、嗍酸酯都不影响反应 进行，三氟甲基化和芳/杂环取代的颈基都可以顺利取 代. 作者通过 ${ }^{31} \mathrm{P}$ NMR 对反应监测的结果推测反应中对 于磺酸酯中氧原子转移的机理和硝基的氧原子转移的 机理类似.

\section{6 烯烃炔烃氢化还原}

烯烃还原是有机合成中重要的反应之一, 早在 1977 年 Walker 课题组 ${ }^{[65-66]}$ 就实现了计量有机膦参与的 烯烃还原反应. 2020 年, Werner 课题组 ${ }^{[67]}$ 报道了首例基 于 $\mathrm{P}(\mathrm{III}) / \mathrm{P}(\mathrm{V})$ 氧化还原循环的催化烯烃还原策略

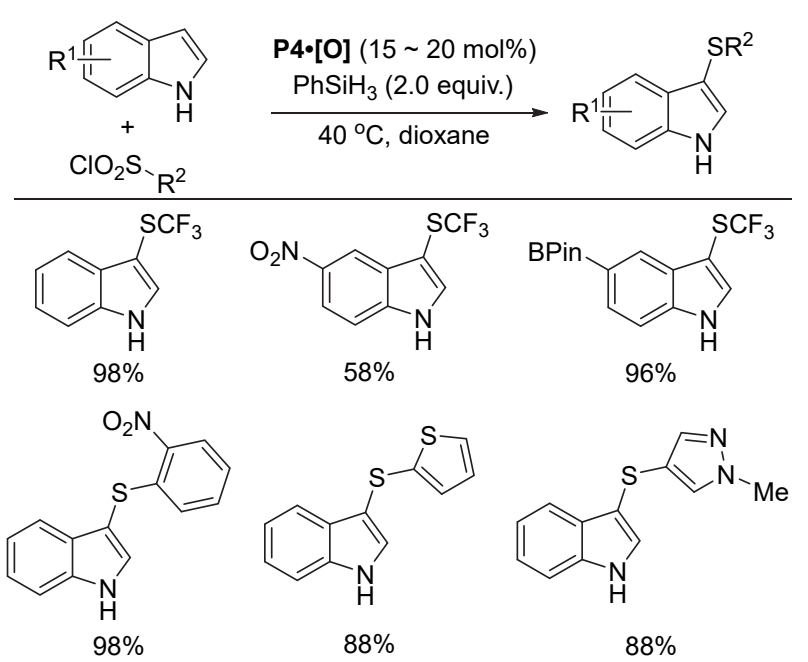

图式 31 膦介导的还原颈基化反应

Scheme 31 Phosphine-mediated reductive sulfhydrylation

(Scheme 32). 通过催化剂前体 P4-[O]和硅烷的使用，有 效地实现了对缺电子双键的选择性还原, 而不影响富电 子双键、硝基等可被还原官能团. 值得一提的是水对这 一过程没有影响. 在反应过程中硅烷还原催化剂前体得 到的膦催化剂 P4 对活泼双键或参键亲核进攻形成两性 离子中间体，随后与水反应形成季鏻盐，接着经过消除 得到产物和 P4・[O], 完成催化循环.

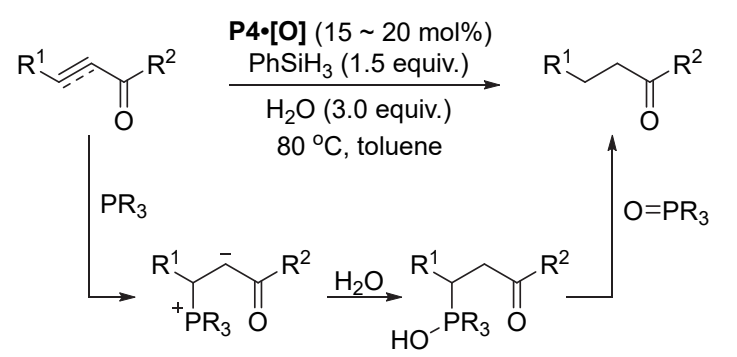<smiles>C=CCOC(=O)CCC(=O)OCC=C</smiles>

图式 32 膦介导的氢化还原反应

Scheme 32 Phosphine-mediated hydrogenation reduction

\section{7 结论与展望}

有机膦化合物介导的 Wittig 反应、Staudinger 反应、 Appel 反应、Mitsunobu 反应在有机合成中早已展现了重 要的价值. 而近来发展的基于 $\mathrm{P}(\mathrm{III}) / \mathrm{P}(\mathrm{V})$ 氧化还原循环 的催化反应更是为这些经典反应带来了新的思考. 通过 引入硅烷、金属氢化物等还原剂, 有效避免了膦氧化物 的生成; 通过高张力催化剂的设计，降低反应能垒，使 得还原更容易进行; 通过特殊骨架催化剂的巧妙设计, 使得 Mitsunobu 反应中膦催化剂无需发生价态变化即可 
完成反应. 关于这些类型的反应模式发展迅速, 充满活 力. 而对于这一领域未来的发展还有以下机遇与挑战:

(1)膦氧化物的还原. 虽然这里介绍的反应都有效 地避免膦氧化物的生成, 但是大多数都引入了更大计量 的硅烷作为还原剂, 无疑增加了生产成本. 而且大部分 反应由于涉及到膦氧化物的还原过程, 需要非常苛刻的 条件. 因此, 对于膦氧化物的还原, 应该发展更廉价更 温和的方法来完成. 比如借助电催化、光催化还原的模 式.

(2)不对称催化反应的设计: 无论是人名反应、新兴 的氧原子迁移反应还是氢化还原反应, 对于不对称反应 的探索仍然有限. 其主要原因是手性催化剂的设计, 一 方面要满足大的张力易于发生氧化还原循环的过程, 另 一方面又要有大的位阻来实现手性的控制.

(3)催化剂的设计: 除了上述提到的从氧化还原循 环的催化剂设计要求, 还应该发展更多的类似于绕开经 典 Mitsunobu 反应机理的催化剂, 实现无需价态变化完 成催化循环。

(4)共催化: 上述反应中有很多有趣的中间体，比如 亚胺基膦可以与亲核性底物发生反应; 膦与硝基发生 $[3+1]$ 环化后的中间体可以串联亲核加成、胺化、 $\mathrm{C}-\mathrm{H}$ 活化等过程, 将其他催化体系合理引入, 也必将会进一 步拓展膦催化的反应模式.

\section{References}

[1] Wittig, G.; Schollkopf, U. Chem. Ber. 1954, 87, 1318 .

[2] Staudinger, H.; Meyer, J. Helv. Chim. Acta 1919, 2, 635.

[3] Appel, R. Angew. Chem., Int. Ed. 1975, 14, 801.

[4] Mitsunobu, O.; Yamada, M. Bull. Chem. Soc. Jpn. 1967, 40, 2380.

[5] Denney, D. B.; DiLeone, R. R. J. Am. Chem. Soc. 1962, 84, 4737.

[6] Denney, D. B.; Jones, D. H. J. Am. Chem. Soc. 1969, 91, 5821

[7] Bartlett, P. D.; Baumstark, A. L.; Landis, M. E. J. Am. Chem. Soc. 1973, 95, 6486

[8] Covitz, F.; Westheimer, F. H. J. Am. Chem. Soc. 1963, 85, 1773.

[9] Naumann, K.; Zon, G.; Mislow, K. J. Am. Chem. Soc. 1969, 91, 7012.

[10] Bryan, M. C.; Dunn, P. J.; Entwistle, D.; Gallou, F.; Koenig, S. G.; Hayler, J. D.; Hickey, M. R.; Hughes, S.; Kopach, M. E.; Moine, G.; Richardson, P.; Roschangar, F.; Steven, A.; Weiberth, F. J. Green Chem. 2018, 20, 5082.

[11] Hérault, D.; Nguyen, D. H.; Nuel, D.; Buono, G. Chem. Soc. Rev. 2015, 44, 2508

[12] O’Brien, C. J.; Tellez, J. L.; Nixon, Z. S.; Kang, L. J.; Carter, A. L.; Kunkel, S. R.; Przeworski, K. C.; Chass, G. A. Angew. Chem., Int. Ed. 2009, 48, 6836.

[13] O’Brien, C. J.; Lavigne, F.; Coyle, E. E.; Holohan, A. J.; Doonan, B. J. Chem. - Eur. J. 2013, 19, 5854.

[14] Coyle, E. E.; Doonan, B. J.; Holohan, A. J.; Walsh, K. A.; Lavigne, F.; Krenske, E. H.; O'Brien, C. J. Angew. Chem., Int. Ed. 2014, 53, 12907.

[15] Cao, J.; Zhou, F.; Zhou, J. Angew. Chem., Int. Ed. 2010, 49, 4976

[16] Longwitz, L.; Spannenberg, A.; Werner, T. ACS Catal. 2019, 9, 9237.

[17] Nykaza, T. V.; Cooper, J. C.; Radosevich, A. T. Org. Synth. 2019, $96,418$.

[18] Hoffmann, M.; Deshmukh, S.; Werner, T. Eur. J. Org. Chem. 2015,
$2015,4532$.

[19] Wang, L.; Sun, M.; Ding, M. W. Eur. J. Org. Chem. 2017, 2017, 2568.

[20] Werner, T.; Hoffmann, M.; Deshmukh, S. Eur. J. Org. Chem. 2014, 2014,6630 .

[21] Werner, T.; Hoffmann, M.; Deshmukh, S. Eur. J. Org. Chem. 2015 , $2015,3286$.

[22] Schirmer, M.-L. L.; Adomeit, S.; Werner, T. Org. Lett. 2015, 17, 3078 .

[23] Schirmer, M.-L.; Adomeit, S.; Spannenberg, A.; Werner, T. Chem. Eur. J. 2016, 22, 2458.

[24] Longwitz, L.; Werner, T. Angew. Chem., Int. Ed. 2020, 59, 2760.

[25] Lee, C. J.; Chang, T. H.; Yu, J. K.; Madhusudhan Reddy, G.; Hsiao, M. Y.; Lin, W. Org. Lett. 2016, 18, 3758.

[26] Saleh, N.; Voituriez, A. J. Org. Chem. 2016, 81, 4371.

[27] Saleh, N.; Blanchard, F.; Voituriez, A. Adv. Synth. Catal. 2017, 359, 2304.

[28] Zhang, K.; Cai, L.; Yang, Z.; Houk, K. N.; Kwon, O. Chem. Sci. 2018, 9, 1867.

[29] Zhang, Q.; Zhu, Y.; Jin, H.; Huang, Y. Chem. Commun. 2017, 53, 3974.

[30] Lorton, C.; Castanheiro, T.; Voituriez, A. J. Am. Chem. Soc. 2019, 141,10142 .

[31] van Kalkeren, H. A.; Bruins, J. J.; Rutjes, F. P. J. T.; van Delft, F. L. Adv. Synth. Catal. 2012, 354, 1417.

[32] Lenstra, D. C.; Wolf, J. J.; Mecinović, J. J. Org. Chem. 2019, 84, 6536.

[33] Kosal, A. D.; Wilson, E. E.; Ashfeld, B. L. Angew. Chem., Int. Ed. 2012, 51,12036

[34] Andrews, K. G.; Denton, R. M. Chem. Commun. 2017, 53, 7982.

[35] White, P. B.; Rijpkema, S. J.; Bunschoten, R. P.; Mecinović, J. Org. Lett. 2019, 21, 1011 .

[36] van Kalkeren, H. A.; Te Grotenhuis, C.; Haasjes, F. S.; Hommersom, C. A.; Rutjes, F. P. J. T.; van Delft, F. L. Eur. J. Org. Chem. 2013, 2013, 7059 .

[37] Wang, L.; Wang, Y.; Chen, M.; Ding, M. W. Adv. Synth. Catal. 2014, 356, 1098 .

[38] Wang, L.; Xie, Y. B.; Huang, N. Y.; Yan, J. Y.; Hu, W. M.; Liu, M. G.; Ding, M. W. ACS Catal. 2016, 6, 4010.

[39] Bel Abed, H.; Mammoliti, O.; Bande, O.; Van Lommen, G.; Herdewijn, P. Org. Biomol. Chem. 2014, 12, 7159.

[40] Lertpibulpanya, D.; Marsden, S. P.; Rodriguez-Garcia, I.; Kilner, C. A. Angew. Chem., Int. Ed. 2006, 45, 5000.

[41] Headley, C. E.; Marsden, S. P. J. Org. Chem. 2007, 72, 7185.

[42] Cai, L.; Zhang, K.; Chen, S.; Lepage, R. J.; Houk, K. N.; Krenske, E. H.; Kwon, O. J. Am. Chem. Soc. 2019, 141, 9537.

[43] van Kalkeren, H. A.; Leenders, S. H. A. M.; Hommersom, C. R. A.; Rutjes, F. P. J. T.; van Delft, F. L. Chem.-Eur. J. 2011, 17, 11290.

[44] Longwitz, L.; Jopp, S.; Werner, T. J. Org. Chem. 2019, 84, 7863.

[45] Lenstra, D. C.; Rutjes, F. P. J. T.; Mecinović, J. Chem. Commun. 2014, 50, 5763 .

[46] Lecomte, M.; Lipshultz, J. M.; Kim-Lee, S. H.; Li, G.; Radosevich, A. T. J. Am. Chem. Soc. 2019, 141, 12507.

[47] O'Brien, C. J. US 8901365, 2014.

[48] Buonomo, J. A.; Aldrich, C. C. Angew. Chem., Int. Ed. 2015, 54, 13041.

[49] Hirose, D.; Gazvoda, M.; Košmrlj, J.; Taniguchi, T. Org. Lett. 2016, $18,4036$.

[50] Beddoe, R. H.; Sneddon, H. F.; Denton, R. M. Org. Biomol. Chem. 2018, 16, 7774.

[51] Beddoe, R. H.; Andrews, K. G.; Magné, V.; Cuthbertson, J. D.; Saska, J.; Shannon-Little, A. L.; Shanahan, S. E.; Sneddon, H. F.; Denton, R. M. Science 2019, 365, 910.

[52] Harris, J. R.; Haynes, M. T.; Thomas, A. M.; Woerpel, K. A. J. Org. Chem. 2010, 75, 5083.

[53] Zhao, W.; Yan, P. K.; Radosevich, A. T. J. Am. Chem. Soc. 2015, $137,616$.

[54] Osman, F. H.; El-Samahy, F. A. Chem. Rev. 2002, 102, 629. 
[55] Cadogan, J. I. G.; Cameron-Wood, M.; Mackie, R. K.; Searle, R. J. G. J. Chem. Soc. 1965, 4831.

[56] Cadogan, J. I. G. Q. Rev., Chem. Soc. 1968, 22, 222.

[57] Cadogan, J. I. G.; Todd, M. J. J. Chem. Soc. C 1969, 0, 2808.

[58] Nykaza, T. V.; Harrison, T. S.; Ghosh, A.; Putnik, R. A.; Radosevich, A. T. J. Am. Chem. Soc. 2017, 139, 6839.

[59] Schoene, J.; Bel Abed, H.; Schmieder, P.; Christmann, M.; Nazaré, M. Chem.-Eur. J. 2018, 24, 9090.

[60] Nykaza, T. V.; Ramirez, A.; Harrison, T. S.; Luzung, M. R.; Radosevich, A. T. J. Am. Chem. Soc. 2018, 140, 3103.

[61] Tsao, M.-L.; Gritsan, N.; James, T. R.; Platz, M. S.; Hrovat, D. A.; Borden, W. T. J. Am. Chem. Soc. 2003, 125, 9343.
[62] Nykaza, T. V.; Cooper, J. C.; Li, G.; Mahieu, N.; Ramirez, A.; Luzung, M. R.; Radosevich, A. T. J. Am. Chem. Soc. 2018, 140, 15200 .

[63] Nykaza, T. V.; Li, G.; Yang, J.; Luzung, M. R.; Radosevich, A. Angew. Chem., Int. Ed. 2020, 59, 4505.

[64] Ghosh, A.; Lecomte, M.; Kim-Lee, S. H.; Radosevich, A. T. Angew. Chem., Int. Ed. 2019, 58, 2864.

[65] Burnett, M. G.; Oswald, T.; Walker, B. J. J. Chem. Soc., Chem. Commun. 1977, 5, 155.

[66] Cao, S.-H.; Zhang, X.-C.; Wei, Y.; Shi, M. Eur. J. Org. Chem. 2011, 2011, 2668.

[67] Longwitz, L.; Werner, T. Angew. Chem., Int. Ed. 2020, 59, 2760.

(Cheng, F.) 